\title{
Spin vortices and vacancies: interactions and pinning on a square lattice
}

\author{
O. Kapikranian* \\ Institute for Condensed Matter Physics, National Academy of Sciences of Ukraine, 79011 Lviv, Ukraine \\ Yu. Holovatch \\ Institute for Condensed Matter Physics, National Academy of Sciences of Ukraine, 79011 Lviv, Ukraine, \\ Institut für Theoretische Physik, Johannes Kepler Universität Linz, 4040 Linz, Austria
}

(Dated: November 20, 2018)

\begin{abstract}
The study gives a decisive answer to the recently risen question about the type and origin of interaction between spin vortices and spin vacancies in $2 D$ spin models. The approach is based on the low-temperature approximation of the $2 D X Y$ model known as the Villain model and does not involve any additional approximations, thus preserving the lattice structure. The exact form of the Hamiltonian describing a system of topological charges and a vacant site supports the attractive type of interaction between the vacancy and the charges.

The quantitative difference between the characteristics of the vortex behavior in the $2 D X Y$ and Villain models due to the different energy of the vortex "cores" in the two models is pointed out. This leads to a conclusion that the interaction between a vortex and a spin vacancy and between a vortex and the antivortex differs quantitatively for small separations in the two mentioned models.
\end{abstract}

PACS numbers: $05.50 .+\mathrm{q} ; 75.10$

\section{INTRODUCTION}

The term spin vortex has become common in theoretical and experimental studies of magnetic materials. It is, in fact, a particular case of a more general class of physical/mathematical objects called topological defects [1, 2].

Although, strictly speaking, topological defects can be defined only in terms of a continuous field, similar formations can be observed in lattice spin models. Moreover, it is the spin vortices that are responsible for the Berezinskii-Kosterlitz-Thouless (BKT) phase transition in the 2D XY model [3-5] (or, speaking more generally, in classical $2 D$ easy-plane magnets).

Most of the theoretical studies of the vortex properties are limited to the low-temperature continuum model proposed by Kosterlitz and Thouless [4] (KT model, hereafter). However, this approach obviously cannot give satisfactory results, when essentially "discrete" phenomena, as the effects induced by a spinless site, are studied. The lack of theoretical studies regarding spin vortices on a lattice and the related problem of spin vortex-spin vacancy interaction is the principal motivation for the present work.

\section{A. Spin vortices}

The $2 D X Y$ model is usually defined as a system of two-component spins $\mathbf{S}_{\mathbf{r}}$ of unit length which states can be represented by a polar coordinate $-\pi<\theta \leq \pi$ : $\mathbf{S}_{\mathbf{r}}=$ $\left(\cos \theta_{\mathbf{r}}, \sin \theta_{\mathbf{r}}\right)$, placed at sites $\mathbf{r}$ of a square lattice, and

*Electronic address: akap@icmp.lviv.ua described by the Hamiltonian

$$
H_{2 D X Y}=J \sum_{\left\langle\mathbf{r}, \mathbf{r}^{\prime}\right\rangle}\left[1-\cos \left(\theta_{\mathbf{r}}-\theta_{\mathbf{r}^{\prime}}\right)\right] .
$$

Close enough to the ground state we have $\theta_{\mathbf{r}}-\theta_{\mathbf{r}^{\prime}} \simeq 0$ or $\pm 2 \pi$ for neighboring sites $\mathbf{r}, \mathbf{r}^{\prime}$.

Generally, considering two neighboring spins at sites $\mathbf{r}$ and $\mathbf{r}^{\prime}$, one can encounter the two situations: $\left|\theta_{\mathbf{r}}-\theta_{\mathbf{r}^{\prime}}\right|<$ $\pi$ and $\left|\theta_{\mathbf{r}}-\theta_{\mathbf{r}^{\prime}}\right|>\pi$ (the situation $\left|\theta_{\mathbf{r}}-\theta_{\mathbf{r}^{\prime}}\right|=\pi$ can be neglected). In order to define spin vortices in the system under consideration, let us introduce the lattice of sites $\mathbf{R}$, dual to the original lattice (the dual lattice is the set of all the centers of elementary cells of the original lattice), and consider only those bonds $\left(\mathbf{R}, \mathbf{R}^{\prime}\right)$ which intersect the bonds $\left(\mathbf{r}, \mathbf{r}^{\prime}\right)$ of the original lattice for which $\left|\theta_{\mathbf{r}}-\theta_{\mathbf{r}^{\prime}}\right|>\pi$.

In order to consider the bonds of interest in a systematic way, let us say that $\mathbf{r}=(x, y), \mathbf{r}^{\prime}=\left(x^{\prime}, y^{\prime}\right)$ define the bond $\left(\mathbf{r}, \mathbf{r}^{\prime}\right)$ if $\mathbf{r}^{\prime}=(x+a, y)$ for a horizontal bond and $\mathbf{r}^{\prime}=(x, y+a)$ for a vertical bond, where $a$ is the lattice spacing. The same rule is imposed for bonds of the dual lattice. Now, we can ascribe to every bond $\left(\mathbf{R}, \mathbf{R}^{\prime}\right)$ a direction defined by the sign of $\theta_{\mathbf{r}}-\theta_{\mathbf{r}^{\prime}}$ of the intersected bond $\left(\mathbf{r}, \mathbf{r}^{\prime}\right):\left(\mathbf{R} \rightarrow \mathbf{R}^{\prime}\right)$ if $\theta_{\mathbf{r}}-\theta_{\mathbf{r}^{\prime}}>\pi$ for a horizontal bond $\left(\mathbf{R}, \mathbf{R}^{\prime}\right)$ or $\theta_{\mathbf{r}^{\prime}}-\theta_{\mathbf{r}}>\pi$ for a vertical bond $\left(\mathbf{R}, \mathbf{R}^{\prime}\right)$, and $\left(\mathbf{R}^{\prime} \rightarrow \mathbf{R}\right)$ in the opposite case. The introduced representation is unique for a given microstate of the spin system (the revers statement is not true, of course).

The most basic structural unit that can be distinguished in the representation we have built is a path $L$ (either straight or steps-like) connecting two sites of the dual lattice, formed by one or several bonds connected together so that their directions comply with some general direction of the path. In the most general case, that path can be either closed or not closed.

While a closed path $L$ represents a trivial situation, the spin configuration with $L$ starting and ending at different 
sites of the dual lattice is of great interest and is called a vortex-antivortex pair (it can be said that the vortex and the antivortex are centered at the ends of the path $L$ ). The above concerns vortices with topological charges \pm 1 ; pairs of vortices with higher values of topological charge can be defined in terms of several paths that start at the vortex and end at the antivortex. Paths that start at the same site but end at different sites correspond to clusters of vortices with different absolute values of charge (for example, +2 and $-1,-1$ ).

The regions around the vortex origins are characterized by significant disorientation of spins and are called "cores". Moderate spin-wave excitations, when $\theta_{\mathbf{r}}-\theta_{\mathbf{r}^{\prime}} \simeq$ $0, \pm 2 \pi$ everywhere except for the vortex "cores", cannot destroy the vortex-antivortex pair unless the two defects annihilate at the same point.

Short-range exchange forces between spins lead to long-range effective interaction between vortices. The energy of this interaction can be explicitly singled out in the Hamiltonian of the Villain model, and turns out to depend only on the essentially inherent characteristic of the vortices called topological charge [6]. It will be shown that the logarithmic asymptotic form, obtained by Villain for the attraction energy of the vortex and the antivortex at large separations, in fact holds sufficiently well on a lattice up to the smallest possible separation of one lattice spacing $a$ (if neglecting the subtle anisotropy effects).

On the contrary, the corresponding energy which we estimate for the $2 D X Y$ model turns out to deviate from the logarithmic law at small separations. In particular, our result for the energy needed to create a vortexantivortex pair is approximately $6.6 J$ ( $J$ is the coupling constant), in contrast to $9.9 \mathrm{~J}$ of the Villain model [6], and in reasonable agreement with the recent results of Monte Carlo simulations [7, 8].

The details of the results announced above can be found in Section $\amalg$

\section{B. Spin vacancies}

An aspect of the spin vortex behavior, which only recently drew attention of the researchers, is the effective interaction with nonmagnetic inclusions in the lattice [911]. Such spin vacancies are part of the models with quenched disorder [12 15] and the lattice gas spin models [16, 17]. Here, we will focus, however, not on the thermodynamic quantities, but on the effective Hamiltonian which describes the interaction between spin vortices and vacancies.

To our knowledge, the first theoretical works devoted to this problem demonstrated global deformation of the vortex structure caused by a single vacancy and repulsive interaction between the vortex origin and the vacancy [9, 12]. This result was essentially caused by an application of the KT continuum model which required representation of the vacancy by a cutout of a finite size in the continuous spin field. Subsequently, the same authors denied this nonphysical result, on the basis of their spin dynamics simulations [11].

The problem was resolved phenomenologically, postulating that the vacancy does not change the vortex structure (or the change is negligible) [11]. Under this assumption, the KT theory led to the attractive interaction which agreed with the results of computer simulations. However, this approach, giving correct qualitative picture, was not able to describe the particular details of the lattice under consideration.

In our study, based on the Villain model, we obtain the effective Hamiltonian describing interaction between spin vortices and spin vacancies on a square lattice.

For example, as it will be shown in this paper, the interaction energy for an individual vortex of topological charge $q$ at point $\mathbf{R}$ and a spin vacancy at $\mathbf{r}$ reads:

$$
E(|\mathbf{r}-\mathbf{R}|)=-(\pi-1) \frac{J q^{2}}{|\mathbf{r}-\mathbf{R}|^{2}}+O\left(|\mathbf{r}-\mathbf{R}|^{-2}\right),
$$

i.e. the vacancy and the vortex attract each other.

Eq. (2) is the asymptotic expression which in fact holds well enough for separations as small as just a few lattice spacings. It will be argued that in the $2 D X Y$ model this energy differs considerably from (2) for small separations $|\mathbf{r}-\mathbf{R}|$. For example, the vortex-on-vacancy pinning energy of the Villain model $E(a)=-(3 \pi-4) J q^{2} \simeq$ $-5.425 J q^{2}$, in contrast to that of the $2 D X Y$ model observed in spin dynamics simulations, $-3.54 J$ [11], and other numerical studies, $-3.178 J$ [10].

The details of the results announced here can be found in Section III

\section{VORTICES IN THE VILLAIN AND $2 D X Y$ MODELS}

\section{A. Topological charges in the Villain model}

Studying the low-temperature properties of the model (11) it would be natural to apply the spin-wave (harmonic) approximation (SWA), i.e. to replace $1-\cos \left(\theta_{\mathbf{r}}-\theta_{\mathbf{r}^{\prime}}\right)$ in (11) with $\frac{1}{2}\left(\theta_{\mathbf{r}}-\theta_{\mathbf{r}^{\prime}}\right)^{2}$. Indeed, this allows to examine many important properties of the low-temperature phase of this model [18 20]. However, the states with $\left|\theta_{\mathbf{r}}-\theta_{\mathbf{r}^{\prime}}\right|>$ $\pi$, which are crucial when considering spin vortices, will have non-physical energy in this case. So, the proper harmonic approximation must be

$$
H_{2 D X Y} \simeq \frac{J}{2} \sum_{\left\langle\mathbf{r}, \mathbf{r}^{\prime}\right\rangle}\left(\theta_{\mathbf{r}}-\theta_{\mathbf{r}^{\prime}}-2 \pi m\left(\theta_{\mathbf{r}}-\theta_{\mathbf{r}^{\prime}}\right)\right)^{2}
$$

with

$$
m\left(\theta_{\mathbf{r}}-\theta_{\mathbf{r}^{\prime}}\right)=\left\{\begin{array}{l}
+1, \theta_{\mathbf{r}}-\theta_{\mathbf{r}^{\prime}}>\pi \\
-1, \theta_{\mathbf{r}}-\theta_{\mathbf{r}^{\prime}}<-\pi \\
0,\left|\theta_{\mathbf{r}}-\theta_{\mathbf{r}^{\prime}}\right|<\pi
\end{array}\right.
$$


At low temperatures, $m\left(\theta_{\mathbf{r}}-\theta_{\mathbf{r}^{\prime}}\right)$ can be considered as independent degrees of freedom taking discrete values $0, \pm 1$. In turn, this leads to the Hamiltonian of the Villain model:

$$
H=\frac{J}{2} \sum_{\left\langle\mathbf{r}, \mathbf{r}^{\prime}\right\rangle}\left(\theta_{\mathbf{r}}-\theta_{\mathbf{r}^{\prime}}-2 \pi m_{\mathbf{r}, \mathbf{r}^{\prime}}\right)^{2}
$$

(obviously, $m_{\mathbf{r}, \mathbf{r}^{\prime}}=-m_{\mathbf{r}^{\prime}, \mathbf{r}}$ ).

Assuming that

$$
\theta_{\mathbf{r}}=\varphi_{\mathbf{r}}+\psi_{\mathbf{r}}
$$

where $\varphi_{\mathbf{r}}$ and $\psi_{\mathbf{r}}$ are chosen so that $\left|\varphi_{\mathbf{r}}-\varphi_{\mathbf{r}^{\prime}}\right|<\pi$ for any pair of spins in the system, i.e one can say that the field $\varphi_{\mathbf{r}}$ is vortexless, and all the vortices are "contained" in $\psi_{\mathbf{r}}$, the Hamiltonian (44) can be written as:

$$
\begin{aligned}
H= & \frac{J}{2} \sum_{\left\langle\mathbf{r}, \mathbf{r}^{\prime}\right\rangle}\left[\left(\varphi_{\mathbf{r}}-\varphi_{\mathbf{r}^{\prime}}\right)^{2}+\left(\psi_{\mathbf{r}}-\psi_{\mathbf{r}^{\prime}}-2 \pi m_{\mathbf{r}, \mathbf{r}^{\prime}}\right)^{2}\right] \\
& +J \sum_{\mathbf{r}} \varphi_{\mathbf{r}} \sum_{\mathbf{u}}\left(\psi_{\mathbf{r}}-\psi_{\mathbf{r}+\mathbf{u}}-2 \pi m_{\mathbf{r}, \mathbf{r}+\mathbf{u}}\right)
\end{aligned}
$$

with $\mathbf{u}=( \pm a, 0),(0, \pm a)$ and lattice spacing $a$.

Following Villain [6] , one can choose $\psi_{\mathbf{r}}\left(\left\{m_{\mathbf{r}, \mathbf{r}^{\prime}}\right\}\right)$ such that $\varphi_{\mathbf{r}}$ and $\psi_{\mathbf{r}}$ decouple in the Hamiltonian, i.e. the last term in (6) vanishes:

$$
\sum_{\mathbf{u}}\left(\psi_{\mathbf{r}}-\psi_{\mathbf{r}+\mathbf{u}}-2 \pi m_{\mathbf{r}, \mathbf{r}+\mathbf{u}}\right)=0 \text { for all } \mathbf{r} .
$$

This is realized when

$$
\begin{aligned}
\psi_{\mathbf{r}}= & \frac{\pi}{2} \sum_{\mathbf{R}}\left\{\left(m_{3,4}-m_{1,2}\right) I_{s c}(x-X, y-Y)\right. \\
& +\left(m_{4,1}-m_{2,3}\right) I_{s c}(y-Y, x-X)+\left(m_{1,2}\right. \\
& \left.\left.-m_{2,3}+m_{3,4}-m_{4,1}\right) I_{s s}(x-X, y-Y)\right\}
\end{aligned}
$$

(see Fig. 1) where $\mathbf{R}$ are sites of the dual lattice, which are situated in the centers of elementary cells of the original lattice, and functions $I_{s c}$ and $I_{s s}$ are given by (A1) and (A2). (The asymptotic properties of $I_{s c}$ and $I_{s s}$ are analyzed in Appendix A) In fact, Eq. (8) is another way of presenting the expression obtained by Villain [6].

In (8), the sum over $\mathbf{R}=(X, Y)$ spans the sites of the dual lattice, while coordinate $\mathbf{r}$ represents a site of the original lattice, therefore, $X-x$ and $Y-y$ can be always presented as $(2 n-1) \frac{a}{2}$, where $n$ is an integer. The short notation

$$
I_{s c(s s)}\left((2 n-1) \frac{a}{2},(2 m-1) \frac{a}{2}\right) \equiv I_{s c(s s)}^{n m}
$$

will be helpful.

Due to the properties: $I_{s c}(-X, Y)=-I_{s c}(X, Y)$, $I_{s c}(X,-Y)=I_{s c}(X, Y), I_{s s}(-X, Y)=-I_{s s}(X, Y)$, $I_{s s}(X, Y)=I_{s s}(Y, X)$, it is enough to define $I_{s c}^{n m}$ and $I_{s s}^{n m}$ only for $n, m$ being positive nonzero integers (natural numbers), thus they can be presented as infinite matrices. In the thermodynamic limit, one has (see Appendix A for the general expression) $I_{s c}^{n m}$

$$
=\left(\begin{array}{ccccc}
\frac{1}{\pi} & \frac{1}{2}-\frac{1}{\pi} & \frac{3}{2}-\frac{13}{3 \pi} & \frac{11}{2}-\frac{17}{\pi} & \cdots \\
-\frac{3}{2}+\frac{5}{\pi} & \frac{1}{3 \pi} & -\frac{3}{2}+\frac{5}{\pi} & -\frac{15}{2}+\frac{119}{5 \pi} & \cdots \\
-\frac{15}{2}+\frac{71}{3 \pi} & \frac{5}{2}-\frac{23}{3 \pi} & \frac{1}{5 \pi} & \frac{5}{2}-\frac{23}{3 \pi} & \cdots \\
-\frac{77}{2}+\frac{121}{\pi} & \frac{35}{2}-\frac{823}{15 \pi} & -\frac{7}{2}+\frac{167}{15 \pi} & \frac{1}{7 \pi} & \cdots \\
\vdots & \vdots & \vdots & \vdots & \ddots
\end{array}\right)
$$

and $I_{s s}^{n m}$

$$
=\left(\begin{array}{ccccc}
\frac{1}{2}-\frac{1}{\pi} & 1-\frac{3}{\pi} & 5-\frac{47}{3 \pi} & 26-\frac{245}{3 \pi} & \cdots \\
1-\frac{3}{\pi} & -\frac{1}{2}+\frac{5}{3 \pi} & -2+\frac{19}{3 \pi} & -13+\frac{613}{15 \pi} & \ldots \\
5-\frac{47}{3 \pi} & -2+\frac{19}{3 \pi} & \frac{1}{2}-\frac{23}{15 \pi} & 3-\frac{47}{5 \pi} & \ldots \\
26-\frac{245}{3 \pi} & -13+\frac{613}{15 \pi} & 3-\frac{47}{5 \pi} & -\frac{1}{2}+\frac{167}{105 \pi} & \ldots \\
\vdots & \vdots & \vdots & \vdots & \ddots
\end{array}\right) .
$$

Note that there was no reason for presenting $I_{s c}^{n m}, I_{s s}^{n m}$ as matrices, other than the convenient visualization.

We have found that the exact values of $\psi_{\mathbf{r}}$, provided by (8), (9), (10) within the vortex core, are quite close to that of its asymptotic form found by Villain [6] :

$$
\psi_{\mathbf{r}} \simeq \sum_{\mathbf{R}} q_{\mathbf{R}} \Phi_{\mathbf{r}}(\mathbf{R}),
$$

where $\Phi_{\mathbf{r}}(\mathbf{R})$ is the polar coordinate of point $\mathbf{r}$ in the coordinate system with its origin at point $\mathbf{R}$ (the reference angles are such that $\psi_{\mathbf{r}}-\psi_{\mathbf{r}^{\prime}}>\pi$ if $m_{\mathbf{r}, \mathbf{r}^{\prime}}=1$, $\psi_{\mathbf{r}}-\psi_{\mathbf{r}^{\prime}}<-\pi$ if $m_{\mathbf{r}, \mathbf{r}^{\prime}}=-1$, and $\left|\psi_{\mathbf{r}}-\psi_{\mathbf{r}^{\prime}}\right|<\pi$ if $\left.m_{\mathbf{r}, \mathbf{r}^{\prime}}=0\right)$, and

$$
q_{\mathbf{R}}=m_{1,2}+m_{2,3}+m_{3,4}+m_{4,1}
$$

is the topological charge defined at site $\mathbf{R}$ of the dual lattice (see Fig. 11).

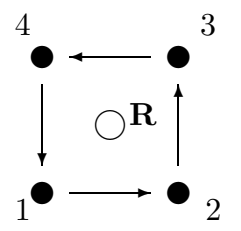

FIG. 1: Plaquette of sites 1,2,3,4 of the initial lattice adjacent to site $\mathbf{R}$ of the dual lattice.

Compare, for example, the field $\psi_{\mathbf{r}}$ given by Eqs. (8) and (11) for a vortex-antivortex pair with the minimal separation (see Fig. 2), shown in Tabs. [and II] .

It is worth mentioning that Eq. (11) can be derived from (8), using the asymptotic form of $I_{s c}$ and $I_{s c}$, Eqs. 


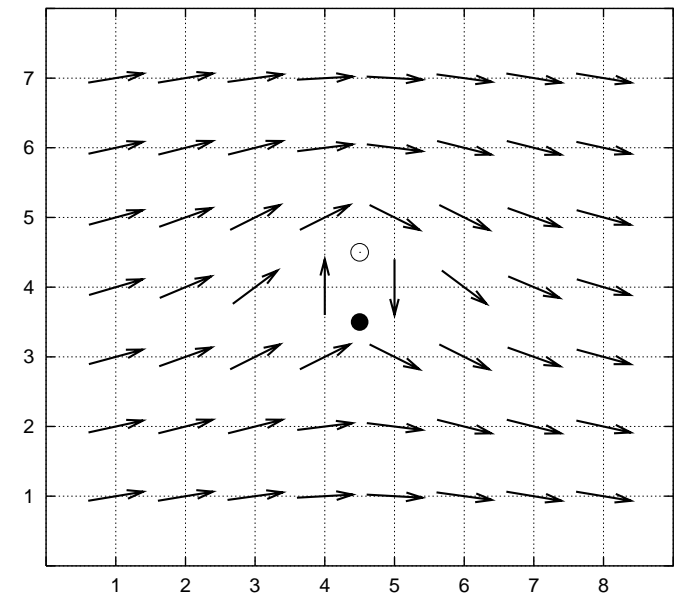

FIG. 2: Field $\psi_{(i, j)}$ for a vortex-antivortex pair situated at sites (4.5.4.5) and (4.5,3.5) (the open and filled circles represent the vortex and the antivortex, respectively) given by Eq. (11) (Tab. (1). The difference with the exact result following from (8) (Tab. III) is insignificant within the resolution of the present picture.

(A9), A10), and integrating (instead of summing over R) along a properly chosen path $L$ (or along several paths for the vortices with higher topological charges, see Section IA) connecting the vortex with its antivortex. We have verified that the form of the field $\psi_{\mathbf{r}}$ given by Eq. (8) is independent of the particular form of this path $L$.

TABLE I: Field $\psi_{(i, j)}$ (see Fig. 20) given by Eq. (11)

\begin{tabular}{c|cccc}
\hline \hline & $i=1$ & $i=2$ & $i=3$ & $i=4$ \\
\hline$j=1$ & 0.1651 & 0.1651 & 0.1355 & 0.0555 \\
$j=2$ & 0.2154 & 0.2450 & 0.2450 & 0.1244 \\
$j=3$ & 0.2630 & 0.3430 & 0.4636 & 0.4636 \\
$j=4$ & 0.2838 & 0.3948 & 0.6435 & $\pi / 2$ \\
\hline \hline
\end{tabular}

TABLE II: Field $\psi_{(i, j)}$ (see Fig. 2) given by Eq. (8)

\begin{tabular}{l|clll}
\hline \hline & $i=1$ & $i=2$ & $i=3$ & $i=4$ \\
\hline \multirow{4}{*}{$j=1$} & $\frac{26}{15}-\frac{\pi}{2}$ & $\frac{26}{15}-\frac{\pi}{2}$ & $\frac{9}{2} \pi-14$ & $\frac{118}{5}-\frac{25}{2} \pi$ \\
& $\simeq 0.1625$ & $\simeq 0.1625$ & $\simeq 0.1372$ & $\simeq 0.0635$ \\
$j=2$ & $\frac{11}{2} \pi-\frac{256}{15}$ & $\frac{\pi}{2}-\frac{4}{3}$ & $\frac{\pi}{2}-\frac{4}{3}$ & $8-\frac{5}{2} \pi$ \\
& $\simeq 0.2121$ & $\simeq 0.2375$ & $\simeq 0.2375$ & $\simeq 0.1460$ \\
& & & & \\
& $\frac{194}{3}-\frac{41}{2} \pi$ & $\frac{34}{3}-\frac{7}{2} \pi$ & $2-\frac{\pi}{2}$ & $2-\frac{\pi}{2}$ \\
& $\simeq 0.2641$ & $\simeq 0.3378$ & $\simeq 0.4292$ & $\simeq 0.4292$ \\
& & & & \\
$j=4$ & $\frac{63}{2} \pi-\frac{296}{3}$ & $\frac{13}{2} \pi-20$ & $\frac{3}{2} \pi-4$ & $\pi / 2$ \\
& $\simeq 0.2934$ & $\simeq 0.4203$ & $\simeq 0.7124$ & $\pi / 2$ \\
\hline \hline
\end{tabular}

\section{B. Interaction between vortices in the Villain and $2 D X Y$ models}

If $\psi_{\mathbf{r}}$ is given by Eq. (8), the Hamiltonian (6) can be reduced to

$$
H=\frac{J}{2} \sum_{\left\langle\mathbf{r}, \mathbf{r}^{\prime}\right\rangle}\left(\varphi_{\mathbf{r}}-\varphi_{\mathbf{r}^{\prime}}\right)^{2}+\sum_{\mathbf{R}, \mathbf{R}^{\prime}} q_{\mathbf{R}} q_{\mathbf{R}^{\prime}} V\left(\mathbf{R}-\mathbf{R}^{\prime}\right),
$$

where topological charge $q_{\mathbf{r}}$ is defined by Eq. (12). Now the vortex interaction energy is given by the second term in the Hamiltonian (13) with

$V\left(\mathbf{R}-\mathbf{R}^{\prime}\right)=\frac{\pi^{2} J}{N} \sum_{\mathbf{k}} \frac{\cos k_{x}\left(X-X^{\prime}\right) \cos k_{y}\left(Y-Y^{\prime}\right)}{\sin ^{2} \frac{k_{x} a}{2}+\sin ^{2} \frac{k_{y} a}{2}}$.

In the thermodynamic limit one can replace the sum over the first Brillouin zone in (14) with an integral, and then, since the difference between the Cartesian coordinates of the vortices centered on sites of the dual lattice is always an integer number of lattice spacing $a$ : $X-X^{\prime}=n a, Y-Y^{\prime}=m a$, following the same scheme of integration which was applied in Appendix A to obtain (A3), (A4), one has:

$$
\begin{aligned}
& V(n a, m a)=\sum_{i=0}^{n} \frac{(-1)^{i}(2 n) !}{(2(n-i)) !(2 i) !} \sum_{j=0}^{m} \frac{(-1)^{j}(2 m) !}{(2(m-j)) !(2 j) !} \\
& \times \sum_{k=0}^{n-i} \frac{(-1)^{k}(n-i) !}{(n-i-k) ! k !} \sum_{l=0}^{m-j} \frac{(-1)^{l}(m-j) !}{(m-j-l) ! l !} F(i+k, j+l)
\end{aligned}
$$

with $F(p, q)$ given by Eq. (A5).

Then the energy of a vortex-antivortex pair, $q_{\mathbf{R}}=+1$ and $q_{\mathbf{R}^{\prime}}=-1$, which follows from (13), is $E_{\text {pair }}(x)=$ $-V(x)$, where $x=\left|\mathbf{R}-\mathbf{R}^{\prime}\right|$ is the distance between the vortex and the antivortex. Comparing $E_{\text {pair }}(x)$ that follows from (15) with the asymptotic expression found by Villain [6]:

$$
E_{\text {pair }}(x) \simeq 10.158 J+2 \pi J \ln (x / a),
$$

see Fig. 3, we notice a fine agreement. The low number of points for small $x / a$ is due to limited number of possibilities to situate the pair on a lattice, and the "oscillation" of data is the anisotropy effect for different orientations of vector $\mathbf{R}-\mathbf{R}^{\prime}$.

As it was already mentioned in the Introduction, the cores of vortices are characterized by large angles between the neighboring spins, so the harmonic approximation (3) cannot give the correct value of the energy of vortex cores in the model (1). Obviously, this can lead to different inter-vortex interaction energies in the Villain and $2 D$ $X Y$ models.

To estimate the energy of the vortex-antivortex interaction in the $2 D X Y$ model, $E_{\text {pair }}^{2 D X Y}(x)$, we consider field

$$
\psi_{\mathbf{r}}^{\prime}=\Phi_{\mathbf{r}}(\mathbf{R})-\Phi_{\mathbf{r}}\left(\mathbf{R}^{\prime}\right)
$$




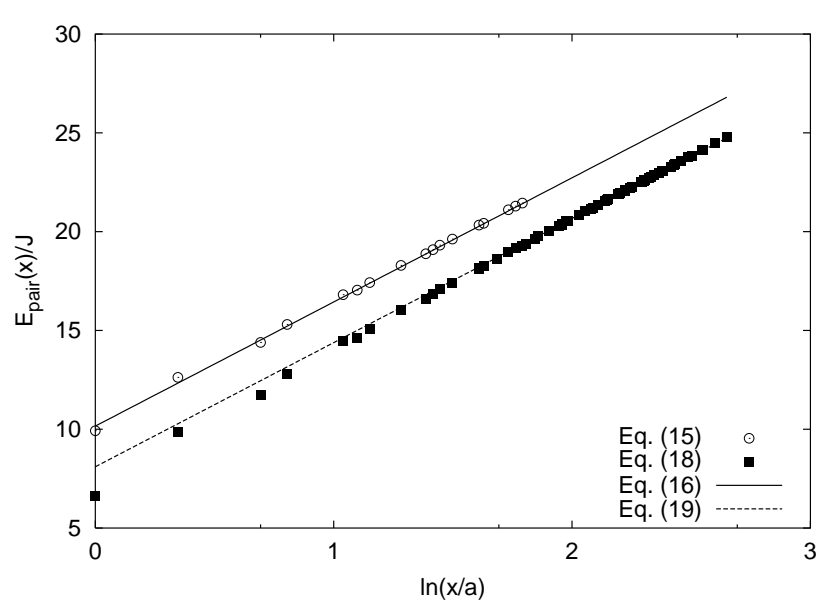

FIG. 3: The energy of a pair of topological charges $q_{\mathbf{R}}=+1$, $q_{\mathbf{R}^{\prime}}=-1$ in the Villain and $2 D X Y$ models as a function of the separation $x=\left|\mathbf{R}-\mathbf{R}^{\prime}\right|$. Open circles represent the exact result for the Villain model, following from (15); filled squares represent the numerical result for the $2 D X Y$ model (see (18)); solid and dashed lines are the asymptotic forms (16) and (19).

(see Eq. (11)), which corresponds to the topological charges $q_{\mathbf{R}}=+1$ and $q_{\mathbf{R}^{\prime}}=-1$, and assume that

$$
E_{\text {pair }}^{2 D X Y}(x)=J \sum_{\left\langle\mathbf{r}, \mathbf{r}^{\prime}\right\rangle}\left[1-\cos \left(\psi_{\mathbf{r}}^{\prime}-\psi_{\mathbf{r}^{\prime}}^{\prime}\right)\right]
$$

performing the summation numerically over a system of sufficiently large size. We are aware that this assumption is not grounded, since $\varphi_{\mathbf{r}}$ and $\psi_{\mathbf{r}}$ cannot be decoupled in the Hamiltonian (11), but it may be instructive.

The quantity which is accessible for measurement in Monte Carlo simulations is the vortex-antivortex pair creation energy in the $2 D X Y$ model, i.e. the energy of a vortex and its antivortex at the minimal separation $a$ (see Fig. 21): $E_{\text {pair }}^{2 D X Y}(a)$. The microcanonical Monte Carlo simulations showed that $E_{\text {pair }}^{2 D X Y}(a) \simeq 7.3 J$ [7], while the canonical MC simulations gave $7.55 \mathrm{~J}$ [8]. Our estimation which follows from (18) is $E_{\text {pair }}^{2 D X Y}(a) \simeq 6.6 J$, in reasonable agreement with the mentioned computer experiments (the exact result for the Villain model is $\pi^{2} J \simeq 9.9 J$, see (15)).

Comparing the result of (18) to the vortex-antivortex interaction energy in the Villain model, see Fig. 3, we see that while at large separations

$$
E_{\text {pair }}^{2 D X Y}(x) \simeq 8.1 J+2 \pi J \ln (x / a)
$$

$E_{\text {pair }}^{2 D X Y}(x)$ deviates considerably from the logarithmic form as the vortex and its antivortex approach each other.

\section{INTERACTION BETWEEN VORTICES AND SPIN VACANCIES}

\section{A. Hamiltonian of the Villain model with spin vacancies}

With the use of variables $c_{\mathbf{r}}$, taking values 1 and 0 depending on whether site $\mathbf{r}$ is occupied with a spin or "empty", respectively, the Hamiltonian of the Villain model with spin vacancies can be presented as [22]:

$$
H=\frac{J}{2} \sum_{\left\langle\mathbf{r}, \mathbf{r}^{\prime}\right\rangle}\left(\theta_{\mathbf{r}}-\theta_{\mathbf{r}^{\prime}}-2 \pi m_{\mathbf{r}, \mathbf{r}^{\prime}}\right)^{2} c_{\mathbf{r}} c_{\mathbf{r}^{\prime}}
$$

Alternatively, it can be written via variables $p_{\mathbf{r}}=1-c_{\mathbf{r}}$ as

$$
H=H_{0}+\sum_{\mathbf{r}} p_{\mathbf{r}} H_{1}(\mathbf{r})+\sum_{\left\langle\mathbf{r}, \mathbf{r}^{\prime}\right\rangle} p_{\mathbf{r}} p_{\mathbf{r}^{\prime}} H_{2}\left(\mathbf{r}, \mathbf{r}^{\prime}\right)
$$

where $H_{0}$ is the Hamiltonian of the Villain model without vacancies (Eq. (20) with all $c_{\mathbf{r}}=1$ ),

$$
H_{1}(\mathbf{r})=-\frac{J}{2} \sum_{\mathbf{u}}\left(\theta_{\mathbf{r}}-\theta_{\mathbf{r}+\mathbf{u}}-2 \pi m_{\mathbf{r}, \mathbf{r}+\mathbf{u}}\right)^{2}
$$

with $\mathbf{u}=( \pm a, 0),(0, \pm a)$ is the change in energy caused by the removal of the four bonds adjacent to the spinless site $\mathbf{r}$, and

$$
H_{2}\left(\mathbf{r}, \mathbf{r}^{\prime}\right)=\frac{J}{2}\left(\theta_{\mathbf{r}}-\theta_{\mathbf{r}^{\prime}}-2 \pi m_{\mathbf{r}, \mathbf{r}^{\prime}}\right)^{2}
$$

compensates the double removal of a common bond of two neighboring sites $\mathbf{r}$ and $\mathbf{r}^{\prime}$ when there happen vacancies on neighboring sites.

Applying (5), one can distinguish in the Hamiltonian (20) terms dependent on vortexless field $\varphi_{\mathbf{r}}$ and/or vortex field $\psi_{\mathbf{r}}$ (marking them with indices $\varphi$ and $\psi$ ):

$$
H=H^{\varphi}+H^{\psi}+H^{\varphi, \psi} .
$$

The first term, considered separately, describes a system of planar spins with angles $\varphi_{\mathbf{r}}$ on a diluted lattice in the SWA, which was the subject of studies [15] and [23], for example. Here, we focus primarily on the last two terms that are connected to the presence of vortices in the system.

Notice that taking $\psi_{\mathbf{r}}$ in the form of Eq. (8) does not lead to decoupling of $\varphi_{\mathbf{r}}$ and $\psi_{\mathbf{r}}$ in the Villain model with spin vacancies $\left(H^{\varphi, \psi} \neq 0\right)$.

\section{B. Hamiltonian of the Villain model with spin vacancies in the Fourier-transformed variables}

Fourier transformation of variables $\varphi_{\mathbf{r}}, \psi_{\mathbf{r}}$ and $m_{\mathbf{r}, \mathbf{r}^{\prime}}$ allows to manipulate Hamiltonian (20) with much ease. The corresponding Fourier transforms $\varphi_{\mathbf{k}}, \psi_{\mathbf{k}}$ and $m_{\mathbf{k}}^{\alpha}$ 
( $\alpha=x, y$ stands to distinguish two sets of Fourier transforms that correspond to "vertical/horizontal" orientation of bond $\left.\left(\mathbf{r}, \mathbf{r}^{\prime}\right)\right)$ can be introduced via the following relations:

$$
\begin{gathered}
\varphi_{\mathbf{k}}=\frac{1}{\sqrt{N}} \sum_{\mathbf{r}} e^{i \mathbf{k r}} \varphi_{\mathbf{r}}, \quad \varphi_{\mathbf{r}}=\frac{1}{\sqrt{N}} \sum_{\mathbf{k}} e^{-i \mathbf{k r}} \varphi_{\mathbf{k}} \\
\psi_{\mathbf{k}}=\frac{1}{\sqrt{N}} \sum_{\mathbf{r}} e^{i \mathbf{k r}} \psi_{\mathbf{r}}, \quad \psi_{\mathbf{r}}=\frac{1}{\sqrt{N}} \sum_{\mathbf{k}} e^{-i \mathbf{k r}} \psi_{\mathbf{k}}, \\
m_{\mathbf{k}}^{\alpha}=\frac{1}{\sqrt{N}} \sum_{\mathbf{r}} e^{i\left(\mathbf{k r}+\frac{k_{\alpha} a}{2}\right)} m_{\mathbf{r}, \mathbf{r}+\mathbf{u}_{\alpha}}, \\
m_{\mathbf{r}, \mathbf{r}+\mathbf{u}_{\alpha}}=\frac{1}{\sqrt{N}} \sum_{\mathbf{k}} e^{-i\left(\mathbf{k r}+\frac{k_{\alpha} a}{2}\right)} m_{\mathbf{k}}^{\alpha}, \quad \alpha=x, y
\end{gathered}
$$

where $N$ is the number of sites in the lattice and the sums over $\mathbf{r}$ and $\mathbf{k}$ span the original lattice and the 1st Brillouin zone of the reciprocal lattice, respectively. Note that in 27) $\mathbf{u}_{x}=(a, 0), \mathbf{u}_{y}=(0, a)$, so the property $m_{\mathbf{r}, \mathbf{r}^{\prime}}=-m_{\mathbf{r}^{\prime}, \mathbf{r}}$ is supposed to be used to obtain the Fourier transform of $m_{\mathbf{r}+\mathbf{u}_{\alpha}, \mathbf{r}}$.
Then, for the field $\psi_{\mathbf{r}}$ given by Eq. (8) one has the Fourier transform [6]

$$
\psi_{\mathbf{k}}=-i \pi \frac{m_{\mathbf{k}}^{x} \sin \frac{k_{x} a}{2}+m_{\mathbf{k}}^{y} \sin \frac{k_{y} a}{2}}{\sin ^{2} \frac{k_{x} a}{2}+\sin ^{2} \frac{k_{y} a}{2}} .
$$

Now, using (28), the condition (7) can be easily checked.

After applying (27) and introducing the Fourier-transform of the topological charge $q_{\mathbf{r}}$ :

$$
q_{\mathbf{k}}=\frac{1}{\sqrt{N}} \sum_{\mathbf{R}} e^{i \mathbf{k} \mathbf{R}} q_{\mathbf{R}}, \quad q_{\mathbf{R}}=\frac{1}{\sqrt{N}} \sum_{\mathbf{k}} e^{-i \mathbf{k} \mathbf{R}} q_{\mathbf{k}}
$$

Eq. (12) takes the form

$$
q_{\mathbf{k}}=2 i\left(m_{\mathbf{k}}^{x} \sin \frac{k_{y} a}{2}-m_{\mathbf{k}}^{y} \sin \frac{k_{x} a}{2}\right) .
$$

Then, it is quite straightforward to obtain (the reader is referred to Eqs. (21) and (24) to understand the upper and bottom indices in the left sides of the equations)

$$
\begin{aligned}
& H_{1}^{\varphi \psi}(\mathbf{r})=\frac{4 \pi J}{N} \sum_{\mathbf{k}} \sum_{\mathbf{k}^{\prime}} \varphi_{\mathbf{k}} q_{\mathbf{k}^{\prime}} \frac{\cos \frac{\left(k_{x}+k_{x}^{\prime}\right) a}{2} \sin \frac{k_{x} a}{2} \sin \frac{k_{y}^{\prime} a}{2}-\cos \frac{\left(k_{y}+k_{y}^{\prime}\right) a}{2} \sin \frac{k_{y} a}{2} \sin \frac{k_{x}^{\prime} a}{2}}{\sin ^{2} \frac{k_{x}^{\prime} a}{2}+\sin ^{2} \frac{k_{y}^{\prime} a}{2}} e^{-i\left(\mathbf{k}+\mathbf{k}^{\prime}\right) \mathbf{r}} \\
& H_{1}^{\psi}(\mathbf{r})=\frac{\pi^{2} J}{N} \sum_{\mathbf{k}} \sum_{\mathbf{k}^{\prime}} q_{\mathbf{k}} q_{\mathbf{k}^{\prime}} \frac{\cos \frac{\left(k_{x}+k_{x}^{\prime}\right) a}{2} \sin \frac{k_{y} a}{2} \sin \frac{k_{y}^{\prime} a}{2}-\cos \frac{\left(k_{y}+k_{y}^{\prime}\right) a}{2} \sin \frac{k_{x} a}{2} \sin \frac{k_{x}^{\prime} a}{2}}{\left(\sin ^{2} \frac{k_{x} a}{2}+\sin ^{2} \frac{k_{y} a}{2}\right)\left(\sin ^{2} \frac{k_{x}^{\prime} a}{2}+\sin ^{2} \frac{k_{y}^{\prime} a}{2}\right)} e^{-i\left(\mathbf{k}+\mathbf{k}^{\prime}\right) \mathbf{r}}, \\
& H_{2}^{\varphi \psi}\left(\mathbf{r}, \mathbf{r}^{\prime}\right)=-\frac{2 \pi J}{N} \sum_{\mathbf{k}} \sum_{\mathbf{k}^{\prime}} \varphi_{\mathbf{k}} q_{\mathbf{k}^{\prime}} e^{-i\left(\mathbf{k}+\mathbf{k}^{\prime}\right) \mathbf{r}}\left(\left(\delta_{\mathbf{r}^{\prime}-\mathbf{r}, \mathbf{u}_{x}} e^{-i \frac{\left(k_{x}+k_{x}^{\prime}\right) a}{2}}+\delta_{\mathbf{r}^{\prime}-\mathbf{r},-\mathbf{u}_{x}} e^{i \frac{\left(k_{x}+k_{x}^{\prime}\right) a}{2}}\right) \sin \frac{k_{x} a}{2} \sin \frac{k_{y}^{\prime} a}{2}\right. \\
& \left.-\left(\delta_{\mathbf{r}^{\prime}-\mathbf{r}, \mathbf{u}_{y}} e^{-i \frac{\left(k_{y}+k_{y}^{\prime}\right) a}{2}}+\delta_{\mathbf{r}^{\prime}-\mathbf{r},-\mathbf{u}_{y}} e^{i \frac{\left(k_{y}+k_{y}^{\prime}\right) a}{2}}\right) \sin \frac{k_{y} a}{2} \sin \frac{k_{x}^{\prime} a}{2}\right)\left(\sin ^{2} \frac{k_{x}^{\prime} a}{2}+\sin ^{2} \frac{k_{y}^{\prime} a}{2}\right)^{-1}, \\
& H_{2}^{\psi}\left(\mathbf{r}, \mathbf{r}^{\prime}\right)=-\frac{\pi^{2} J}{2 N} \sum_{\mathbf{k}} \sum_{\mathbf{k}^{\prime}} q_{\mathbf{k}} q_{\mathbf{k}^{\prime}} e^{-i\left(\mathbf{k}+\mathbf{k}^{\prime}\right) \mathbf{r}}\left(\left(\delta_{\mathbf{r}^{\prime}-\mathbf{r}, \mathbf{u}_{x}} e^{-i \frac{\left(k_{x}+k_{x}^{\prime}\right) a}{2}}+\delta_{\mathbf{r}^{\prime}-\mathbf{r},-\mathbf{u}_{x}} e^{i \frac{\left(k_{x}+k_{x}^{\prime}\right) a}{2}}\right) \sin \frac{k_{y} a}{2} \sin \frac{k_{y}^{\prime} a}{2}\right. \\
& \left.-\left(\delta_{\mathbf{r}^{\prime}-\mathbf{r}, \mathbf{u}_{y}} e^{-i \frac{\left(k_{y}+k_{y}^{\prime}\right) a}{2}}+\delta_{\mathbf{r}^{\prime}-\mathbf{r},-\mathbf{u}_{y}} e^{i \frac{\left(k_{y}+k_{y}^{\prime}\right) a}{2}}\right) \sin \frac{k_{x} a}{2} \sin \frac{k_{x}^{\prime} a}{2}\right) / \sum_{\alpha=x, y} \sin ^{2} \frac{k_{\alpha} a}{2} \sum_{\alpha=x, y} \sin ^{2} \frac{k_{\alpha}^{\prime} a}{2} .
\end{aligned}
$$

\section{Attractive interaction between spin vortices and a spin vacancy}

Returning to variables $\varphi_{\mathbf{r}}$ and $q_{\mathbf{R}}$ in Eqs. (31), (32), one has

$$
\begin{aligned}
H_{1}^{\varphi \psi}(\mathbf{r})= & \pi J \sum_{\mathbf{R}} q_{\mathbf{R}}\left\{\left(\varphi_{\mathbf{r}+\mathbf{u}_{x}}-\varphi_{\mathbf{r}-\mathbf{u}_{x}}\right) I_{s c}(y-Y, x-X)-\left(\varphi_{\mathbf{r}+\mathbf{u}_{y}}-\varphi_{\mathbf{r}-\mathbf{u}_{y}}\right) I_{s c}(x-X, y-Y)\right. \\
& \left.+\left(\varphi_{\mathbf{r}+\mathbf{u}_{y}}+\varphi_{\mathbf{r}-\mathbf{u}_{y}}-\varphi_{\mathbf{r}+\mathbf{u}_{x}}-\varphi_{\mathbf{r}-\mathbf{u}_{x}}\right) I_{s s}(x-X, y-Y)\right\}
\end{aligned}
$$


and

$$
\begin{aligned}
H_{1}^{\psi}(\mathbf{r})= & -\pi^{2} J \sum_{\mathbf{R}} \sum_{\mathbf{R}^{\prime}} q_{\mathbf{R}} q_{\mathbf{R}^{\prime}}\left\{I_{\mathrm{sc}}(x-X, y-Y) I_{\mathrm{sc}}\left(x-X^{\prime}, y-Y^{\prime}\right)+I_{\mathrm{sc}}(y-Y, x-X) I_{\mathrm{sc}}\left(y-Y^{\prime}, x-X^{\prime}\right)\right. \\
& \left.+2 I_{\mathrm{ss}}(x-X, y-Y) I_{\mathrm{ss}}\left(x-X^{\prime}, y-Y^{\prime}\right)\right\},
\end{aligned}
$$

where $I_{s c}$ and $I_{s s}$ are defined by Eqs. (A1) and A2. Analogous expressions for (33) and (34) can be obtained easily.

In order to obtain the effective Hamiltonian describing interaction between vacancies and topological charges only, one has to integrate out $\varphi_{\mathbf{r}}$ in the partition function

$$
Z=\operatorname{Tr}_{\varphi, \psi} e^{-\beta\left(H^{\varphi}+H^{\psi}+H^{\varphi, \psi}\right)},
$$

so that

$$
Z=\operatorname{Tr}_{\psi} e^{-\beta H_{\text {eff }}^{\psi}}
$$

where $H_{\mathrm{eff}}^{\psi}$ is the desired Hamiltonian.

We have to restrict our consideration to the case of one spin vacancy at site $\mathbf{r}^{*}$ to be able to use the results of Appendix B] Then, using (B2) and (31), one has the effective Hamiltonian

$$
\begin{aligned}
H_{\mathrm{eff}}^{\psi}\left(\mathbf{r}^{*}\right)= & H_{1}^{\varphi \psi}\left(\mathbf{r}^{*}\right)+\frac{\pi^{2} J}{N} \sum_{\mathbf{k}, \mathbf{k}^{\prime}} q_{\mathbf{k}} q_{\mathbf{k}^{\prime}}\left[(\pi-2)\left(\sin \frac{k_{x} a}{2} \cos \frac{k_{y} a}{2} \sin \frac{k_{x}^{\prime} a}{2} \cos \frac{k_{y}^{\prime} a}{2}+\sin \frac{k_{y} a}{2} \cos \frac{k_{x} a}{2} \sin \frac{k_{y}^{\prime} a}{2} \cos \frac{k_{x}^{\prime} a}{2}\right)\right. \\
& \left.-2 \frac{4-\pi}{\pi-2} \sin \frac{k_{x} a}{2} \sin \frac{k_{y} a}{2} \sin \frac{k_{x}^{\prime} a}{2} \sin \frac{k_{y}^{\prime} a}{2}\right] e^{-i\left(\mathbf{k}+\mathbf{k}^{\prime}\right) \mathbf{r}^{*}} / \sum_{\alpha=x, y} \sin ^{2} \frac{k_{\alpha} a}{2} \sum_{\alpha=x, y} \sin ^{2} \frac{k_{\alpha}^{\prime} a}{2} .
\end{aligned}
$$

Finally, using (36) and (29), one can write:

$$
\begin{aligned}
H_{\mathrm{eff}}^{\psi}\left(\mathbf{r}^{*}\right)= & -\pi^{2} J \sum_{\mathbf{R}} \sum_{\mathbf{R}^{\prime}} q_{\mathbf{R}} q_{\mathbf{R}^{\prime}}\left\{( \pi - 1 ) \left[I_{\mathrm{sc}}\left(x^{*}-X, y^{*}-Y\right) I_{\mathrm{sc}}\left(x^{*}-X^{\prime}, y^{*}-Y^{\prime}\right)\right.\right. \\
& \left.\left.+I_{\mathrm{sc}}\left(y^{*}-Y, x^{*}-X\right) I_{\mathrm{sc}}\left(y^{*}-Y^{\prime}, x^{*}-X^{\prime}\right)\right]+\frac{4}{\pi-2} I_{\mathrm{ss}}\left(x^{*}-X, y^{*}-Y\right) I_{\mathrm{ss}}\left(x^{*}-X^{\prime}, y^{*}-Y^{\prime}\right)\right\}
\end{aligned}
$$

While (A3) and (A4) provide the exact value of (40) for a discrete lattice, it is instructive to find its asymptotic form:

$$
\begin{aligned}
& H_{\mathrm{eff}}^{\psi}\left(\mathbf{r}^{*}\right)=-(\pi-1) J a^{2} \sum_{\mathbf{R}, \mathbf{R}^{\prime}} q_{\mathbf{R}} q_{\mathbf{R}^{\prime}} \\
& \times\left[\frac{\left(\mathbf{r}^{*}-\mathbf{R}\right)\left(\mathbf{r}^{*}-\mathbf{R}^{\prime}\right)}{\left|\mathbf{r}^{*}-\mathbf{R}\right|^{2}\left|\mathbf{r}^{*}-\mathbf{R}^{\prime}\right|^{2}}+O\left(\frac{1}{\left|\mathbf{r}^{*}-\mathbf{R}\right|^{2}\left|\mathbf{r}^{*}-\mathbf{R}^{\prime}\right|^{2}}\right)\right],
\end{aligned}
$$

which follows from (A9) and A10). If, for example, one has a vortex of topological charge either + or -1 and a spin vacancy, separated by distance $x$, (41) gives the energy of their interaction:

$$
E(x)=-J(\pi-1) a^{2} / x^{2}+O\left(1 / x^{2}\right)
$$

(compare it to the exact result following from Eq. (40) shown in Fig. (4).

\section{A vortex pinned by the vacancy}

An analogue of the condition (77) for the field $\psi_{\mathbf{r}}$, which would assure that $H^{\varphi, \psi}=0$ in the diluted Villain model (20), reads as

$$
c_{\mathbf{r}} \sum_{\mathbf{u}}\left(\psi_{\mathbf{r}}-\psi_{\mathbf{r}+\mathbf{u}}-2 \pi m_{\mathbf{r}, \mathbf{r}+\mathbf{u}}\right) c_{\mathbf{r}+\mathbf{u}}=0 \text { for all } \mathbf{r} .
$$

Numerical studies of spin vortices in the presence of a spinless site [10], 11] suggest that it is energetically preferable for a vortex to be pinned (centered) on the vacancy. Thus, one can assume that

$$
\widetilde{\psi}_{\mathbf{r}}= \pm \Phi_{\mathbf{r}}\left(\mathbf{r}^{*}\right),
$$

where $\Phi_{\mathbf{r}}\left(\mathbf{r}^{*}\right)$ was defined after Eq. (11) and $\mathbf{r}^{*}$ is the coordinate of the vacancy, might satisfy (43) when the topological charge $q= \pm 1$ is on one of the four dual lattice sites $\mathbf{R}^{*}$ adjacent to $\mathbf{r}^{*}$ (see Fig. (5)). 


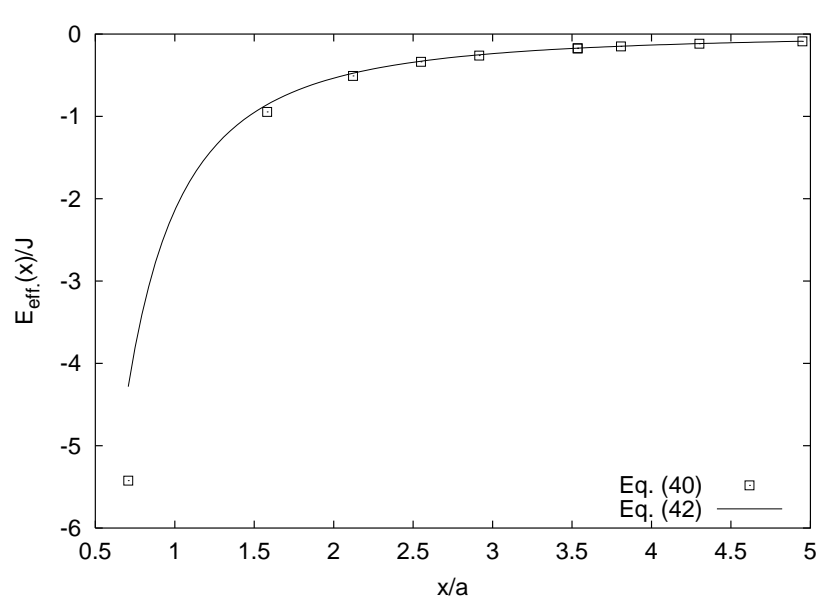

FIG. 4: Interaction energy of a vortex of charge \pm 1 and a vacancy as a function of their separation $x$. Open squares represent the exact result (40) and the solid curve represents the asymptotic expression (42).

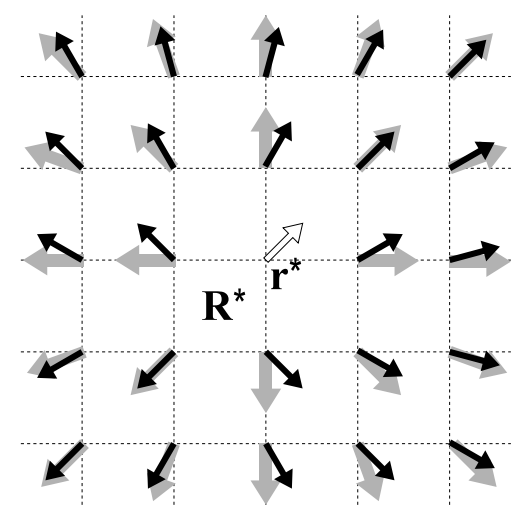

FIG. 5: Representation field $\psi_{\mathbf{r}}$ of topological charge $q=$ +1 situated at site $\mathbf{R}^{*}$ of the dual lattice, which leads to its decoupling in the Hamiltonian of the pure Villain model (black and white arrows) and a model with a vacancy at site $\mathbf{r}^{*}$ (grey arrows).

Then, the vortex-on-vacancy pinning energy, i.e. the energy of the vortex centered on $\mathbf{r}^{*}$ minus that of the vortex centered on $\mathbf{R}^{*}$, can be estimated by numerical summation over a lattice of sufficiently large size:

$$
\begin{aligned}
& E_{\text {pin }}=\frac{J}{2} \sum_{\left\langle\mathbf{r}, \mathbf{r}^{\prime}\right\rangle} {\left[\left(\widetilde{\psi}_{\mathbf{r}}-\widetilde{\psi}_{\mathbf{r}^{\prime}}-2 \pi m_{\mathbf{r}, \mathbf{r}^{\prime}}\right)^{2}\right.} \\
&\left.-\left(\psi_{\mathbf{r}}^{\prime}-\psi_{\mathbf{r}^{\prime}}^{\prime}-2 \pi m_{\mathbf{r}, \mathbf{r}^{\prime}}\right)^{2}\right],
\end{aligned}
$$

where $\psi_{\mathbf{r}}^{\prime}= \pm \Phi_{\mathbf{r}}\left(\mathbf{R}^{*}\right)$, which gives $E_{\text {pin }} \simeq-5.22 J$.

The corresponding energy that follows from (40) is $E_{\text {pin }}=-(3 \pi-4) J \simeq-5.42 J$. The difference from the result of (45) is not surprising, if one notices that $\widetilde{\psi}_{\mathbf{r}}$ only approximately fulfills (43) for almost all the lattice sites.

It is worth mentioning that using the exchange potential of the $2 D X Y$ model, Eq. (11), numerical summation analogous to that of (45) leads to $E_{\text {pin }}^{2 D X Y} \simeq-3.21 J$, which agrees with $-3.178 \mathrm{~J}$ of the energy minimizing iterative method [10] and $-3.54 \mathrm{~J}$ of the spin dynamics simulations [11].

\section{CONCLUSIONS}

The exact and asymptotic expressions for the interaction energy of topological charges and a spinless site, Eqs. (40) and (41), found for the Villain model on a square lattice, definitively confirm the attractive character of the interaction. This agrees with the results of the spin dynamics simulations for the $2 D X Y$ model [11] and the energy minimizing iterative method for the easy-plane Heisenberg model [10].

However, we showed that this interaction in the $2 D$ $X Y$ model can differ from Eq. (40), that corresponds to the Villain model, considerably at small separations due to different energies of the vortex "cores" (regions with strong disorientation of spins). In particular, the exact value of the vortex-on-vacancy pinning energy in the Villain model, $E_{\text {pin }}=-(3 \pi-4) J \simeq-5.42 J$, differs significantly from that found in [11] and [10] $(-3.54 J$ and $-3.178 J$, respectively).

Moreover, we showed that the mentioned difference of the vortex cores' energies in the two models leads to a deviation of the vortex-antivortex interaction energy in the $2 D X Y$ model from a logarithmic law at small separations, while the corresponding energy of the Villain model retains logarithmic dependence on separation $x$ (if we neglect slight anisotropy effects) up to the smallest possible distance on a lattice which is of one lattice spacing, $x=a$.

We have estimated the vortex-antivortex pair creation energy for the $2 D X Y$ model as $E_{\text {pair }}^{2 D X Y}(a) \simeq 6.6 J$ (in contrast to $\pi^{2} J \simeq 9.9 J$ of the Villain model), which is in reasonable agreement with the results of the recent Monte Carlo simulations [8], 7] (7.55J and 7.3J, respectively).

\section{Acknowledgments}

We thank Prof. Bertrand Berche for his useful corrections to the manuscript, and for the discussions we have had. We wish to acknowledge the support of cooperation programme "Dnipro" between the Ministry of Foreign Affairs of France and the Ministry of Education and Science of Ukraine. Yu. H. was supported in part by the Austrian Fonds zur Förderung der wissenschaftlichen Forschung under Project No. P19583-N20. 


\section{Appendix A: Functions $I_{s c}(X, Y)$ and $I_{s s}(X, Y)$}

In this appendix we study the functions

$$
\begin{aligned}
& I_{s c}(X, Y)=\frac{1}{N} \sum_{\mathbf{k}} \frac{\sin \frac{k_{x} a}{2} \cos \frac{k_{y} a}{2}}{\sum_{\alpha=x, y} \sin ^{2} \frac{k_{\alpha} a}{2}} \sin k_{x} X \cos k_{y} Y, \\
& I_{s s}(X, Y)=\frac{1}{N} \sum_{\mathbf{k}} \frac{\sin \frac{k_{x} a}{2} \sin \frac{k_{y} a}{2}}{\sum_{\alpha=x, y} \sin ^{2} \frac{k_{\alpha} a}{2}} \sin k_{x} X \sin k_{y} Y,
\end{aligned}
$$

which enter many important expressions concerning the behavior of topological charges, and the sums over $\mathbf{k}$ in (A1), (A2) span the first Brillouin zone.

For $X=(2 n-1) a, Y=(2 m-1) a(n, m=1,2,3, \ldots)$ (A1) and A2 can be calculated exactly, replacing the sums with integrals in the thermodynamic limit. The integration gives

$$
\begin{aligned}
I_{s c}((2 n-1) a,(2 m-1) a)= & \sum_{i=0}^{n-1} \frac{(-1)^{i}(2 n-1) !}{(2(n-i-1)) !(2 i+1) !} \sum_{j=0}^{m-1} \frac{(-1)^{j}(2 m-1) !}{(2(m-j)-1) !(2 j) !} \sum_{k=0}^{n-i-1} \frac{(-1)^{k}(n-i-1) !}{(n-i-k-1) ! k !} \\
& \times \sum_{l=0}^{m-j} \frac{(-1)^{l}(m-j) !}{(m-j-l) ! l !} F(i+k+1, j+l) \\
I_{s s}((2 n-1) a,(2 m-1) a)= & \sum_{i=0}^{n-1} \frac{(-1)^{i}(2 n-1) !}{(2(n-i-1)) !(2 i+1) !} \sum_{j=0}^{m-1} \frac{(-1)^{j}(2 m-1) !}{(2(m-j-1)) !(2 j+1) !} \sum_{k=0}^{n-i-1} \frac{(-1)^{k}(n-i-1) !}{(n-i-k-1) ! k !} \\
& \times \sum_{l=0}^{m-j-1} \frac{(-1)^{l}(m-j-1) !}{(m-j-l-1) ! l !} F(i+k+1, j+l+1)
\end{aligned}
$$

with

$$
\begin{aligned}
F(p, q)= & \sum_{u=0}^{q-1}(-1)^{u} \frac{(2(p+u)-1) ! !}{(2(p+u)) ! !} \frac{(2(q-u-1)-1) ! !}{(2(q-u-1)) ! !}+\frac{1}{2} \sum_{u=0}^{p+q-1} \frac{(-1)^{q+u}(p+q-1) !(2 u-1) ! !}{(p+q-u-1) !(u !)^{2}} \\
& -\frac{1}{\pi} \sum_{u=1}^{p+q-1} \frac{(-1)^{q+u}(p+q-1)}{(p+q-u-1) ! u !}\left(\frac{(2 u-1) ! !}{u !} \sum_{w=1}^{u-1} \frac{(u-w-1) !}{(2(u-w)-1) ! !}+\frac{1}{u}\right) .
\end{aligned}
$$

These results were obtained by expressing $\sin k_{x} X$, $\sin k_{y} Y, \cos k_{y} Y$ as polynomials $P\left(\sin \frac{k_{\alpha} a}{2}, \cos \frac{k_{\alpha} a}{2}\right)$, and then applying the standard tables of integrals [25]. We used the notations: $(2 n) ! ! \equiv \prod_{i=1}^{n} 2 i,(2 n-1) ! ! \equiv$ $\prod_{i=1}^{n}(2 i-1)$; when $n=0:(2 n) ! ! \equiv 1,(2 n-1) ! ! \equiv 1$. The sums of no meaning, like $\sum_{i=n}^{m}$ with $m<n$, that may be encountered in fal for some values of $p, q$, should be interpreted as equal to zero.

It is instructive to find an asymptotic form for (A1) and (A2). It turns out that simple analytic expressions can be obtained, assuming that at least one of the arguments $X, Y$ is large. Using the integral [24]

$$
\int_{0}^{\infty} \frac{\cos x}{x^{2}+a^{2}}=\frac{\pi}{2|a|} e^{-|a|}
$$

one can show that

$$
\begin{aligned}
I_{s c}(X \rightarrow \infty, Y)= & \frac{a}{\pi} \int_{0}^{\pi / a} d k_{y} e^{-X \frac{2}{a} \sin \frac{k_{y} a}{2}} \cos k_{y} Y \\
& \times \sinh \left(\sin \frac{k_{y} a}{2}\right) \cot \frac{k_{y} a}{2} \\
= & \frac{a}{\pi} \int_{0}^{\pi / a} d k_{y} e^{-X k_{y}} \cos k_{y} Y
\end{aligned}
$$

and

$$
\begin{gathered}
I_{s c}(X, Y \rightarrow \infty)=\frac{a}{\pi} \int_{0}^{\pi / a} d k_{x} e^{-Y \frac{2}{a} \sin \frac{k_{x} a}{2}} \cos k_{x} X \\
\times \cosh \left(\sin \frac{k_{x} a}{2}\right)=\frac{a}{\pi} \int_{0}^{\pi / a} d k_{y} e^{-Y k_{x}} \sin k_{x} X .
\end{gathered}
$$


So,

$$
I_{s c}(X, Y)=\frac{a}{\pi} \frac{X}{X^{2}+Y^{2}},
$$

when at least one of the arguments $X, Y$ is sufficiently large.

In a similar way one can show that

$$
I_{s s}(X, Y)=\frac{a^{2}}{\pi} \frac{X Y}{\left(X^{2}+Y^{2}\right)^{2}},
$$

if at least one of the arguments $X, Y$ is sufficiently large.

\section{Appendix B: Hamiltonian describing topological charges in a system with a spin vacancy}

The aim of the present appendix is to show how the "vortexless" degrees of freedom $\varphi_{\mathbf{r}}$ can be integrated out in the partition function (37) when only one spin vacancy at site $\mathbf{r}^{*}$ is considered, so that (see Subsection $\amalg$ A )

$$
c_{\mathbf{r}}=\left\{\begin{array}{ll}
0, & \mathbf{r}=\mathbf{r}^{*}, \\
1, & \mathbf{r} \neq \mathbf{r}^{*} ;
\end{array} \quad \text { or } \quad p_{\mathbf{r}}= \begin{cases}1, & \mathbf{r}=\mathbf{r}^{*} \\
0, & \mathbf{r} \neq \mathbf{r}^{*}\end{cases}\right.
$$

As we will show below, the partition function can be presented in this case in the form (38) with

$$
\begin{aligned}
& H_{\mathrm{eff}}=H_{\psi}-\frac{1}{4 \beta^{2} J}\left(\sum_{\mathbf{k}} \frac{\eta_{\mathbf{k}} \eta_{-\mathbf{k}}}{\gamma_{\mathbf{k}}}\right. \\
& -\frac{\pi}{4(\pi-2)} \frac{1}{N} \sum_{\mathbf{k}, \mathbf{k}^{\prime}}\left(g_{\mathbf{k},-\mathbf{k}^{\prime}}+g_{\mathbf{k}, \mathbf{k}^{\prime}}\right) \frac{\eta_{-\mathbf{k}} \eta_{-\mathbf{k}^{\prime}}}{\gamma_{\mathbf{k}^{\prime}}} e^{-i\left(\mathbf{k}+\mathbf{k}^{\prime}\right) \mathbf{r}^{*}} \\
& \left.+\frac{\pi}{4} \frac{1}{N} \sum_{\mathbf{k}, \mathbf{k}^{\prime}}\left(g_{\mathbf{k},-\mathbf{k}^{\prime}}+g_{\mathbf{k}, \mathbf{k}^{\prime}}\right) \frac{\eta_{-\mathbf{k}} \eta_{-\mathbf{k}^{\prime}}}{\gamma_{\mathbf{k}^{\prime}}} e^{-i\left(\mathbf{k}+\mathbf{k}^{\prime}\right) \mathbf{r}^{*}}\right),(\mathrm{B} 2
\end{aligned}
$$

where $\gamma_{\mathbf{k}} \equiv 2\left(\sin ^{2} \frac{k_{x} a}{2}+\sin ^{2} \frac{k_{y} a}{2}\right)$,

$$
g_{\mathbf{k}, \mathbf{k}^{\prime}} \equiv\left(\gamma_{\mathbf{k}+\mathbf{k}^{\prime}}-\gamma_{\mathbf{k}}-\gamma_{\mathbf{k}^{\prime}}\right) / \gamma_{\mathbf{k}}
$$

and

$$
\eta_{\mathbf{k}} \equiv \frac{\beta J}{\sqrt{N}} e^{-i \mathbf{k r}^{*}} \sum_{\mathbf{u}} e^{-i \mathbf{k u}}\left(\psi_{\mathbf{r}^{*}+\mathbf{u}}-\psi_{\mathbf{r}^{*}}-2 \pi m_{\mathbf{r}^{*}+\mathbf{u}, \mathbf{r}^{*}}\right) .
$$

\section{The partition function of the Villain model on the lattice with a spin vacancy}

Let us denote

$$
Z_{\psi} \equiv \operatorname{Tr}_{\varphi} e^{-\beta\left(H_{\varphi}+H_{\varphi, \psi}\right)},
$$

So

$$
Z=\operatorname{Tr}_{\psi}\left(e^{-\beta H_{\psi}} Z_{\psi}\right)
$$

Using Fourier transformation (25), one can rewrite the terms that depend on $\varphi_{\mathbf{r}}$ in the Hamiltonian (24) as (see [23]):

$H_{\varphi}=J \sum_{\mathbf{k}} \gamma_{\mathbf{k}} \varphi_{\mathbf{k}} \varphi_{-\mathbf{k}}+\frac{J}{N} \sum_{\mathbf{k}, \mathbf{k}^{\prime}} e^{-i\left(\mathbf{k}+\mathbf{k}^{\prime}\right) \mathbf{r}^{*}} g_{\mathbf{k}, \mathbf{k}^{\prime}} \varphi_{\mathbf{k}} \varphi_{\mathbf{k}^{\prime}}$

where $g_{\mathbf{k}, \mathbf{k}^{\prime}}$ was defined in (B3) and the sums are over the 1st Brillouin zone. Correspondingly, the mixed $\varphi \psi$-term in Eq. (24) reads:

$$
\begin{aligned}
H_{\varphi, \psi}= & -\frac{J}{\sqrt{N}} \sum_{\mathbf{k}}\left(\varphi_{\mathbf{k}} e^{-i \mathbf{k} \mathbf{r}^{*}}\right. \\
& \left.\times \sum_{\mathbf{u}} e^{-i \mathbf{k u}}\left(\psi_{\mathbf{r}^{*}+\mathbf{u}}-\psi_{\mathbf{r}^{*}}-2 \pi m_{\mathbf{r}^{*}+\mathbf{u}, \mathbf{r}^{*}}\right)\right) .
\end{aligned}
$$

Using the Taylor series expansion, $Z_{\psi}$ can be written as:

$$
\begin{aligned}
Z_{\psi}= & \operatorname{Tr}_{\varphi} e^{-\beta J \sum_{\mathbf{k}} \gamma_{\mathbf{k}} \varphi_{\mathbf{k}} \varphi_{-\mathbf{k}}+\sum_{\mathbf{k}} \eta_{\mathbf{k}} \varphi_{\mathbf{k}}} \\
& \times\left(1+\sum_{n=1}^{\infty} \frac{1}{n !} I_{\left(\varphi_{\mathbf{k}_{1}}, \varphi_{\mathbf{k}_{2}}\right), \ldots\left(\varphi_{\mathbf{k}_{2 n-1}, \varphi_{\mathbf{k}_{2 n}}}\right)}\right)
\end{aligned}
$$

where $\eta_{\mathbf{k}}$ was defined in (B4) and

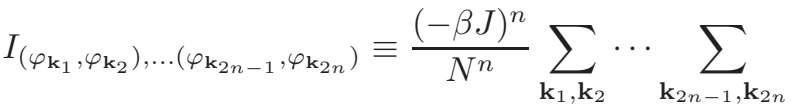

$$
\begin{aligned}
& \times e^{-i\left(\mathbf{k}_{1}+\ldots+\mathbf{k}_{2 n}\right) \mathbf{r}^{*}} g_{\mathbf{k}_{1}, \mathbf{k}_{2}} \cdots g_{\mathbf{k}_{2 n-1}, \mathbf{k}_{2 n}} \varphi_{\mathbf{k}_{1}} \cdots \varphi_{\mathbf{k}_{2 n}} .
\end{aligned}
$$

Now, introducing the notations:

$$
Z_{*} \equiv \operatorname{Tr}_{\varphi} e^{-\beta J \sum_{\mathbf{k}} \gamma_{\mathbf{k}} \varphi_{\mathbf{k}} \varphi_{-\mathbf{k}}+\sum_{\mathbf{k}} \eta_{\mathbf{k}} \varphi_{\mathbf{k}}}
$$

and

$$
\langle\ldots\rangle_{*} \equiv Z_{*}^{-1} \operatorname{Tr}_{\varphi}\left(e^{-\beta J \sum_{\mathbf{k}} \gamma_{\mathbf{k}} \varphi_{\mathbf{k}} \varphi_{-\mathbf{k}}+\sum_{\mathbf{k}} \eta_{\mathbf{k}} \varphi_{\mathbf{k}}} \ldots\right),
$$

(B9) becomes

$$
Z_{\psi}=Z_{*}\left(1+\sum_{n=1}^{\infty} \frac{1}{n !}\left\langle I_{\left(\varphi_{\mathbf{k}_{1}}, \varphi_{\mathbf{k}_{2}}\right), \ldots\left(\varphi_{\mathbf{k}_{2 n-1}}, \varphi_{\mathbf{k}_{2 n}}\right)}\right\rangle_{*}\right) .
$$

\section{Calculation of $Z_{*}$ and $\left\langle\varphi_{\mathbf{k}_{1}} \ldots \varphi_{\mathbf{k}_{2 n}}\right\rangle_{*}$}

$Z_{*}$ and $\left\langle\varphi_{\mathbf{k}_{1}} \ldots \varphi_{\mathbf{k}_{2 n}}\right\rangle_{*}$ are the first quantities to be calculated.

Since $\varphi_{\mathbf{k}}\left(\right.$ for $\mathbf{k} \neq 0$ ) is a complex quantity: $\varphi_{\mathbf{k}}=$ $\varphi_{\mathbf{k}}^{c}+i \varphi_{\mathbf{k}}^{s}, \operatorname{Tr}_{\varphi}$ should be understood as:

$$
\operatorname{Tr}_{\varphi}=\prod_{\mathbf{k} \in B_{1 / 2}} \int_{-\infty}^{\infty} d \varphi_{\mathbf{k}}^{c} \int_{-\infty}^{\infty} d \varphi_{\mathbf{k}}^{s}
$$

where $B_{1 / 2}$ stands for a half of the 1st Brillouin zone excluding $\mathbf{k}=0\left(\varphi_{\mathbf{k}}^{c}\right.$ and $\varphi_{\mathbf{k}}^{s}$ in the other half are not 
independent, due to the relations: $\varphi_{-\mathbf{k}}^{c}=\varphi_{\mathbf{k}}^{c}$ and $\varphi_{-\mathbf{k}}^{s}=$ $\left.-\varphi_{\mathbf{k}}^{s}\right)$. It was possible to extend the bounds of integration to infinity in (B14) and omit writing the integral over $\varphi_{0}$, since the functions that stand after the trace in our calculations are always rapidly decaying when $\beta J \rightarrow \infty$ and independent from $\varphi_{0}$.

Then, it is straightforward to obtain

$$
Z_{*}=\left(\prod_{\mathbf{k} \neq 0} \sqrt{\frac{\pi}{2 \beta J \gamma_{\mathbf{r}}}}\right) e^{\frac{1}{4 \beta J} \sum_{\mathbf{k} \neq 0} \frac{\eta_{\mathbf{k}} \eta_{-\mathbf{k}}}{\gamma_{\mathbf{k}}}}
$$

Using (B15), it is easy to show that

$$
\left\langle\varphi_{\mathbf{k}_{1}} \ldots \varphi_{\mathbf{k}_{2 n}}\right\rangle_{*}=Z_{*}^{-1} 2^{-2 n} \frac{\partial}{\partial \eta_{\mathbf{k}_{1}}} \cdots \frac{\partial}{\partial \eta_{\mathbf{k}_{2 n}}} Z_{*},
$$

where

$$
\frac{\partial}{\partial \eta_{\mathbf{k}}} \equiv \frac{\partial}{\partial \eta_{\mathbf{k}}^{c}}-i \frac{\partial}{\partial \eta_{\mathbf{k}}^{s}}, \quad \frac{\partial}{\partial \eta_{-\mathbf{k}}} \equiv \frac{\partial}{\partial \eta_{\mathbf{k}}^{c}}+i \frac{\partial}{\partial \eta_{\mathbf{k}}^{s}} .
$$

Noting that $\frac{\partial \eta_{\mathbf{k}}}{\partial \eta_{\mathbf{k}^{\prime}}}=2 \delta_{\mathbf{k}, \mathbf{k}^{\prime}}\left(\delta_{\mathbf{k}, \mathbf{k}^{\prime}}\right.$ is Kronecker delta), one arrives at

$$
\begin{aligned}
\left\langle\varphi_{\mathbf{k}_{1}} \ldots \varphi_{\mathbf{k}_{2 n}}\right\rangle_{*}= & \sum_{l=0}^{n} \frac{1}{(2 \beta J)^{2 n-l}} \sum_{\text {pairs } 2 n \rightarrow l} \\
& \times \prod_{u=1}^{l} \frac{\delta_{\mathbf{k}_{i_{u}},-\mathbf{k}_{j_{u}}}}{\gamma_{\mathbf{k}_{i_{u}}}} \prod_{w=1}^{2 n-2 l} \frac{\eta-\mathbf{k}_{p_{w}}}{\gamma_{\mathbf{k}_{p_{w}}}}
\end{aligned}
$$

where the sum $\sum_{\text {pairs } 2 n \rightarrow l}$ spans all the possible ways of selecting $l$ indistinguishable unordered pairs $\left(i_{u}, j_{u}\right), u=$ $1, \ldots, l$ out of $2 n$ indexes $1, \ldots, 2 n$. (It is easy to see that

$$
\left.\sum_{\text {pairs } 2 n \rightarrow l} 1=\frac{(2 n) !}{l !(2 !)^{l}(2 n-2 l) !} \cdot\right)
$$

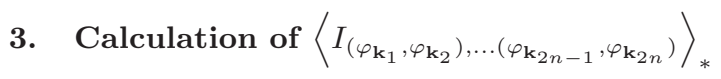

According to (B10) and (B12),

$$
\begin{gathered}
\left\langle I_{\left(\varphi_{\mathbf{k}_{1}}, \varphi_{\mathbf{k}_{2}}\right), \ldots\left(\varphi_{\mathbf{k}_{2 n-1}}, \varphi_{\mathbf{k}_{2 n}}\right)}\right\rangle_{*}=\frac{(-\beta J)^{n}}{N^{n}} \sum_{\mathbf{k}_{1}, \ldots \mathbf{k}_{2 n}} \\
\times e^{-i\left(\mathbf{k}_{1}+\ldots+\mathbf{k}_{2 n}\right) \mathbf{r}^{*}} g_{\mathbf{k}_{1}, \mathbf{k}_{2}} \cdots g_{\mathbf{k}_{2 n-1}, \mathbf{k}_{2 n}}\left\langle\varphi_{\mathbf{k}_{1}} \cdots \varphi_{\mathbf{k}_{2 n}}\right\rangle_{*} .
\end{gathered}
$$

At this stage, it is convenient to introduce the notions:

$$
\begin{aligned}
I_{i} \equiv & \frac{1}{N^{i}} \sum_{\mathbf{k}_{1}, \ldots, \mathbf{k}_{i}} g_{\mathbf{k}_{1},-\mathbf{k}_{2}} g_{\mathbf{k}_{2},-\mathbf{k}_{3}} \cdots g_{\mathbf{k}_{i-1},-\mathbf{k}_{i}} g_{\mathbf{k}_{i},-\mathbf{k}_{1}} \\
I_{i}^{*} \equiv & \frac{1}{N^{i}} \sum_{\mathbf{k}_{1}, \ldots, \mathbf{k}_{i+1}} g_{\mathbf{k}_{1},-\mathbf{k}_{2}} g_{\mathbf{k}_{2},-\mathbf{k}_{3}} \cdots g_{\mathbf{k}_{i-1},-\mathbf{k}_{i}} g_{\mathbf{k}_{i}, \mathbf{k}_{i+1}} \\
& \times e^{-i\left(\mathbf{k}_{1}+\mathbf{k}_{i+1}\right) \mathbf{r}^{*}} \frac{\eta_{\mathbf{k}_{1}} \eta_{\mathbf{k}_{i+1}}}{\gamma_{\mathbf{k}_{i+1}}}
\end{aligned}
$$

Then, insertion of (B18) into (B19) leads to a polynomial form with respect to $I_{i}$ and $I_{i}^{*}(i=1, \ldots, \infty)$ :

$$
\begin{aligned}
& \left\langle I_{\left(\varphi_{\mathbf{k}_{1}}, \varphi_{\mathbf{k}_{2}}\right), \ldots\left(\varphi_{\mathbf{k}_{2 n-1}}, \varphi_{\mathbf{k}_{2 n}}\right)}\right\rangle_{*}=(-1)^{n} \sum_{l=0}^{n} \frac{2^{-n}}{(2 \beta J)^{n-l}} \\
& \times\left(\prod_{i=1}^{l} \sum_{\lambda_{i}=0}^{[l / i]}\right)\left(\prod_{j=1}^{n-l} \sum_{\lambda_{j}^{*}=0}^{[(n-l) / j]}\right) \delta\left(\sum_{i=1}^{l} i \lambda_{i}-l\right) \\
& \times \delta\left(\sum_{j=1}^{n-l} j \lambda_{j}^{*}-(n-l)\right) \Lambda_{\lambda_{1}, \ldots, \lambda_{l}}^{\lambda_{1}^{*}, \ldots, \lambda_{n-l}^{*}} I_{1}^{\lambda_{1}} \ldots I_{l}^{\lambda_{l}} \\
& \times\left(I_{1}^{*}\right)^{\lambda_{1}^{*}} \ldots\left(I_{n-l}^{*}\right)^{\lambda_{n-l}^{*}},
\end{aligned}
$$

where $[a]$ means the nearest integer not exceeding $a$,

$$
\begin{aligned}
& \delta(x)=\left\{\begin{array}{l}
1, x=0 \\
0, x \neq 0
\end{array},\right. \text { and } \\
& \quad \Lambda_{\lambda_{1}, \ldots, \lambda_{l}}^{\lambda_{1}^{*}, \ldots, \lambda_{n-l}^{*}}=n ! \prod_{i=1}^{l} \frac{\left[2^{i-1}(i-1) !\right]^{\lambda_{i}}}{\lambda_{i} !(i !)^{\lambda_{i}}} \prod_{j=1}^{n-l} \frac{\left[2^{j-1} j !\right]^{\lambda_{j}^{*}}}{\lambda_{i}^{*} !(i !)^{\lambda_{i}^{*}}}
\end{aligned}
$$

is the combinatorial "weight" given by the number of ways of selecting $\lambda_{1}$ unordered elements, $\lambda_{2}$ unordered groups of two unordered elements, ..., $\lambda_{l}$ unordered groups of $l$ unordered elements, $\lambda_{1}^{*}$ unordered elements, $\lambda_{2}^{*}$ unordered groups of two unordered elements, ..., and $\lambda_{n-l}^{*}$ unordered groups of $n-l$ unordered elements out of $n$ distinct elements, which is

$$
\begin{array}{r}
n ! /\left(\lambda_{1} ! \lambda_{2} ! \cdots \lambda_{l} ! \lambda_{1}^{*} ! \lambda_{2}^{*} ! \cdots \lambda_{n-l}^{*} !(1 !)^{\lambda_{1}}(2 !)^{\lambda_{2}} \cdots(l !)^{\lambda_{l}}\right. \\
\left.(1 !)^{\lambda_{1}^{*}}(2 !)^{\lambda_{2}^{*}} \cdots((n-l) !)^{\lambda_{n-l}^{*}}\right),
\end{array}
$$

times the number of distinct ways of connecting 4 distinct elements belonging to 2 distinct groups, each consisting of two elements, with 2 indistinguishable links in such a manner that the two elements of one group are connected to the elements belonging to another group, raised to the power $\lambda_{2}$, times the product over $i=\overline{1, l}$ of the number of distinct ways of connecting $2 i$ distinct elements belonging to $i$ distinct groups, each consisting of two elements, with $i$ indistinguishable links in such a manner that the two elements of each group are connected to the elements belonging to two another groups, raised to the power $\lambda_{i}$, i.e.

$$
\times \prod_{i=1}^{l}\left[2^{i-1}(i-1) !\right]^{\lambda_{i}}
$$

times the number of distinct ways of connecting 4 distinct elements belonging to 2 distinct groups, each consisting of two elements, with 1 link in such a manner that one of the two elements of one group is connected to one of the two elements belonging to another group, raised to the power $\lambda_{2}^{*}$, times the product over $j=\overline{1, n-l}$ of the number of distinct ways of connecting $2 j$ distinct elements belonging to $j$ distinct groups, each consisting of two elements, with $j-1$ indistinguishable links in such 
a manner that one of the two elements of any group is connected to one of the two elements of another group and the second element is either connected to one of the two elements of a different group or not connected, raised to the power $\lambda_{j}^{*}$, i.e.

$$
\times \prod_{j=1}^{n-l}\left[2^{j-1} j !\right]^{\lambda_{j}^{*}}
$$

Inserting (B23) into (B22), one has

$$
\begin{aligned}
& \left\langle I_{\left(\varphi_{\mathbf{k}_{1}}, \varphi_{\mathbf{k}_{2}}\right), \ldots\left(\varphi_{\mathbf{k}_{2 n-1}}, \varphi_{\mathbf{k}_{2 n}}\right)}\right\rangle_{*}=(-1)^{n} n ! \sum_{l=0}^{n} \frac{1}{(2 \beta J)^{n-l}} \\
& \times \prod_{i=1}^{l} \sum_{\lambda_{i}=0}^{[l / i]} \frac{1}{\lambda_{i} !}\left(\frac{I_{i}}{2 i}\right)^{\lambda_{i}} \prod_{j=1}^{n-l} \sum_{\lambda_{j}^{*}=0}^{[(n-l) / j]} \frac{1}{\lambda_{i}^{*} !}\left(\frac{I_{i}^{*}}{2}\right)^{\lambda_{i}^{*}} \\
& \times \delta\left(\sum_{i=1}^{l} i \lambda_{i}-l\right) \delta\left(\sum_{j=1}^{n-l} j \lambda_{j}^{*}-(n-l)\right),
\end{aligned}
$$

and then, inserting (B26) in (B13), one can notice that the infinite series in (B13) can be rearranged as it is shown below:

$$
\begin{aligned}
Z_{\psi}= & Z_{*} \prod_{i=1}^{\infty}\left(1+(-1)^{i} \frac{I_{i}}{2 i}+\frac{1}{2 !}\left((-1)^{i} \frac{I_{i}}{2 i}\right)^{2}\right. \\
& \left.+\frac{1}{3 !}\left((-1)^{i} \frac{I_{i}}{2 i}\right)^{3}+\ldots\right) \prod_{j=1}^{\infty}\left(1+(-1)^{j} \frac{I_{j}^{*}}{4 \beta J}\right. \\
& \left.+\frac{1}{2 !}\left((-1)^{j} \frac{I_{j}^{*}}{4 \beta J}\right)^{2}+\frac{1}{3 !}\left((-1)^{j} \frac{I_{j}^{*}}{4 \beta J}\right)^{3}+\ldots\right),
\end{aligned}
$$

or

$$
\begin{aligned}
Z_{\psi}= & Z_{*} \exp \left(\frac{1}{2} \sum_{i=1}^{\infty}(-1)^{i} I_{i} / i\right) \\
& \times \exp \left(\frac{1}{4 \beta J} \sum_{j=1}^{\infty}(-1)^{j} I_{j}^{*}\right)
\end{aligned}
$$

\section{Calculation of $I_{i}$ and $I_{i}^{*}$}

Eqs. (B20) and (B21) can be written as

$$
I_{i}=\frac{1}{N} \sum_{\mathbf{k}} \tilde{I}_{i-1}(\mathbf{k},-\mathbf{k})
$$

and

$$
I_{i}^{*}=\frac{1}{N} \sum_{\mathbf{k}, \mathbf{k}^{\prime}} \tilde{I}_{i-1}\left(\mathbf{k}, \mathbf{k}^{\prime}\right) \frac{\eta_{-\mathbf{k}} \eta_{-\mathbf{k}^{\prime}}}{\gamma_{\mathbf{k}^{\prime}}} e^{-i\left(\mathbf{k}+\mathbf{k}^{\prime}\right) \mathbf{r}^{*}}
$$

$(i \geq 1)$ with

$$
\tilde{I}_{i}\left(\mathbf{k}, \mathbf{k}^{\prime}\right) \equiv \frac{1}{N^{i}} \sum_{\mathbf{k}_{1}, \ldots, \mathbf{k}_{i}} g_{\mathbf{k},-\mathbf{k}_{1}} g_{\mathbf{k}_{1},-\mathbf{k}_{2}} \cdots g_{\mathbf{k}_{i-1},-\mathbf{k}_{i}} g_{\mathbf{k}_{i}, \mathbf{k}^{\prime}}
$$

for $i \geq 1$ and $\tilde{I}_{0}\left(\mathbf{k}, \mathbf{k}^{\prime}\right) \equiv g_{\mathbf{k}, \mathbf{k}^{\prime}}$. One can notice the obvious recurrent relation

$$
\tilde{I}_{i+1}\left(\mathbf{k}, \mathbf{k}^{\prime}\right)=\frac{1}{N} \sum_{\mathbf{k}^{*}} \tilde{I}_{i}\left(\mathbf{k},-\mathbf{k}^{*}\right) g_{\mathbf{k}^{*}, \mathbf{k}^{\prime}}
$$

In the thermodynamic limit, one can replace the sum $\frac{1}{N} \sum_{\mathbf{k}}$ over the 1st Brillouin zone by the integrals $\frac{a^{2}}{(2 \pi)^{2}} \int_{-\pi / a}^{\pi / a} d k_{x} \int_{-\pi / a}^{\pi / a} d k_{y}$, and then, noticing that

$$
\frac{a^{2}}{\pi^{2}} \int_{0}^{\pi / a} d k_{x} \int_{0}^{\pi / a} d k_{y} \frac{\sin ^{4} \frac{k_{x} a}{2}}{\sin ^{2} \frac{k_{x} a}{2}+\sin ^{2} \frac{k_{y} a}{2}}=\frac{1}{\pi}
$$

and

$$
\begin{array}{r}
\frac{a^{2}}{\pi^{2}} \int_{0}^{\pi / a} d k_{x} \int_{0}^{\pi / a} d k_{y} \frac{\sin ^{2} \frac{k_{x} a}{2} \cos ^{2} \frac{k_{x} a}{2}}{\sin ^{2} \frac{k_{x} a}{2}+\sin ^{2} \frac{k_{y} a}{2}}=\frac{a^{2}}{\pi^{2}} \\
\times \int_{0}^{\pi / a} d k_{x} \int_{0}^{\pi / a} d k_{y} \frac{\sin ^{2} \frac{k_{x} a}{2} \sin ^{2} \frac{k_{y} a}{2}}{\sin ^{2} \frac{k_{x} a}{2}+\sin ^{2} \frac{k_{y} a}{2}}=\frac{1}{2}-\frac{1}{\pi},
\end{array}
$$

one can show that

$$
\begin{gathered}
\frac{1}{N} \sum_{\mathbf{k}^{\prime}} g_{\mathbf{k},-\mathbf{k}^{\prime}} g_{\mathbf{k}^{\prime}, \mathbf{k}^{\prime \prime}}=\left(1-\frac{2}{\pi}\right) g_{\mathbf{k},-\mathbf{k}^{\prime \prime}} \\
-\frac{1}{\pi}\left(g_{\mathbf{k},-\mathbf{k}^{\prime \prime}}+g_{\mathbf{k}, \mathbf{k}^{\prime \prime}}\right)+\left(\frac{1}{2}-\frac{1}{\pi}\right) \gamma_{\mathbf{k}^{\prime \prime}}, \\
\frac{1}{N} \sum_{\mathbf{k}^{\prime}} g_{\mathbf{k}, \mathbf{k}^{\prime}} g_{\mathbf{k}^{\prime}, \mathbf{k}^{\prime \prime}}=\left(1-\frac{2}{\pi}\right) g_{\mathbf{k}, \mathbf{k}^{\prime \prime}} \\
-\frac{1}{\pi}\left(g_{\mathbf{k},-\mathbf{k}^{\prime \prime}}+g_{\mathbf{k}, \mathbf{k}^{\prime \prime}}\right)+\left(\frac{1}{2}-\frac{1}{\pi}\right) \gamma_{\mathbf{k}^{\prime \prime}} .
\end{gathered}
$$

and

$$
\frac{1}{N} \sum_{\mathbf{k}} \gamma_{\mathbf{k}} g_{\mathbf{k}, \mathbf{k}^{\prime}}=-\gamma_{\mathbf{k}^{\prime}}
$$

Then, it is easy to see that:

$$
\tilde{I}_{i}\left(\mathbf{k}, \mathbf{k}^{\prime}\right)=A_{i} g_{\mathbf{k},(-1)^{i} \mathbf{k}^{\prime}}+B_{i}\left(g_{\mathbf{k},-\mathbf{k}^{\prime}}+g_{\mathbf{k}, \mathbf{k}^{\prime}}\right)+C_{i} \gamma_{\mathbf{k}^{\prime}}
$$

with coefficients $A_{i}, B_{i}$ and $C_{i}$, obeying the recurrent relations:

$$
\begin{gathered}
A_{i+1}=\left(1-\frac{2}{\pi}\right) A_{i} \\
B_{i+1}=-\frac{1}{\pi} A_{i}+\left(1-\frac{4}{\pi}\right) B_{i}, \\
C_{i+1}=\left(\frac{1}{2}-\frac{1}{\pi}\right)\left(A_{i}+2 B_{i}\right)-C_{i},
\end{gathered}
$$

and $A_{0}=1, B_{0}=0, C_{0}=0$. Thus,

$$
A_{i}=\left(1-\frac{2}{\pi}\right)^{i}
$$




$$
\begin{aligned}
B_{i} & =-\frac{1}{\pi} \sum_{j=0}^{i-1}\left(1-\frac{4}{\pi}\right)^{j}\left(1-\frac{2}{\pi}\right)^{i-1-j} \\
& =-\frac{1}{2}\left[\left(1-\frac{2}{\pi}\right)^{i}-\left(1-\frac{4}{\pi}\right)^{i}\right], \\
C_{i} & =(-1)^{i-1}\left(\frac{1}{2}-\frac{1}{\pi}\right) \sum_{j=0}^{i-1}(-1)^{j}\left(1-\frac{4}{\pi}\right)^{j} \\
& =\frac{1}{4}\left[(-1)^{i-1}+\left(1-\frac{4}{\pi}\right)^{i}\right] .
\end{aligned}
$$

Finally, one can obtain expressions for $I_{i}$ and $I_{i}^{*}$ and check that

$$
\begin{aligned}
& \sum_{i=1}^{\infty}(-1)^{i} I_{i}^{*}=-\frac{\pi}{4(\pi-2)} \\
& \times \frac{1}{N} \sum_{\mathbf{k}, \mathbf{k}^{\prime}}\left(g_{\mathbf{k},-\mathbf{k}^{\prime}}+g_{\mathbf{k}, \mathbf{k}^{\prime}}\right) \frac{\eta_{-\mathbf{k}} \eta_{-\mathbf{k}^{\prime}}}{\gamma_{\mathbf{k}^{\prime}}} e^{-i\left(\mathbf{k}+\mathbf{k}^{\prime}\right) \mathbf{r}^{*}} \\
& +\frac{\pi}{4} \frac{1}{N} \sum_{\mathbf{k}, \mathbf{k}^{\prime}}\left(g_{\mathbf{k},-\mathbf{k}^{\prime}}+g_{\mathbf{k}, \mathbf{k}^{\prime}}\right) \frac{\eta_{-\mathbf{k}} \eta_{-\mathbf{k}^{\prime}}}{\gamma_{\mathbf{k}^{\prime}}} e^{-i\left(\mathbf{k}+\mathbf{k}^{\prime}\right) \mathbf{r}^{*}}
\end{aligned}
$$

In conclusion, using (B6), (B15), (B27), and (B32), we obtain (B2).
[1] N. D. Mermin, Rev. Mod. Phys. 51, 591 (1979)

[2] P. M. Chaikin, T. C. Lubenskii, Principles of condensed matter physics (Cambridge University Press, Cambridge, 1995)

[3] V. L. Berezinskii, Sov. Phys. J.E.T.P. 34610 (1972)

[4] J. M. Kosterlitz, D. J. Thouless, J. Phys. C 6, 1181 (1973)

[5] J. M. Kosterlitz, J. Phys. C 7, 1046 (1974)

[6] J. Villain, J. Phys. (Paris) 36, 581 (1975)

[7] S. B. Ota, S. Ota, Phys. Lett. A 206133 (1995)

[8] R. Gupta and C. F. Baillie, Phys. Rev. B 452883 (1992)

[9] L. A. S. Mól, A. R. Pereira, A. S. T. Pires, Phys. Rev. B 66, $052415(2002)$

[10] G. M. Wysin, Phys. Rev. B 68184411 (2003)

[11] A. R. Pereira, L. A. S. Mól, S. A. Leonel, P. Z. Coura, B. V. Costa, Phys. Rev. B 68, 132409 (2003)

[12] S. A. Leonel, P. Z. Coura, A. R. Pereira, L. A. S. Mól, B. V. Costa, Phys. Rev. B 67, 104426 (2003)

[13] G. M. Wysin, A. R. Pereira, I. A. Marques, S. A. Leonel, P. Z. Coura, Phys. Rev. B 72, 094418 (2005)

[14] G. M. Wysin, Phys. Rev. B 71, 094423 (2005)

[15] B. Berche, A. I. Fariñas-Sánchez, Yu. Holovatch, R. Paredes V., Eur. Phys. J. B 36, 91 (2003)
[16] H. Chamati, S. Romano, Phys. Rev. B 73, 184424 (2006)

[17] H. Chamati, S. Romano, Phys. Rev. B 75, 184413 (2007)

[18] F. Wegner, Z. Phys. 206, 465 (1967)

[19] J. Tobochnik and G. V. Chester, Phys. Rev. B 20, 3761 (1979)

[20] S. T. Bramwell, J.-Y. Fortin, P. C. W. Holdsworth, S. Peysson, J.-F. Pinton, B. Portelli, M. Sellitto, Phys. Rev. E 63, 041106 (2001)

[21] J. V. José, L. P. Kadanoff, S. Kirkpatrick, D. A. Nelson, Phys. Rev. B 16, 1217 (1977)

[22] O. Kapikranian, B. Berche, Yu. Holovatch, Phys. Lett.A 372, 5716 (2008)

[23] O. Kapikranian, B. Berche, and Yu. Holovatch, Eur. Phys. J. B 5693 (2007)

[24] R. J. Tallarida, Pocket book of integrals and mathematical formulas (3rd ed., Chapman \& Hall/CRC, Boca Raton, London, New York, Washington, D.C., 1999)

[25] A. P. Prudnikov, Yu. A. Brychkov, O. I. Marichev, Integrals and series: Volume 1: Elementary functions (CRC Press, 1986) 